\title{
Continuous blood pressure monitoring with MMW radar sensor
}

\author{
Ryota Kawasaki $^{1}$, Akihiro Kajiwara ${ }^{1 \text { a) }}$ \\ ${ }^{1}$ Graduate School of Environment Engineering, University of Kitakyushu \\ 1-1, Hibikino, Wakamatuku-ku, Kitakyushu, Fukuoka 808-0135, Japan \\ a)kajiwara@kitakyu-u.ac.jp
}

\begin{abstract}
Blood pressure (BP) is one of the four main vital signs providing valuable medical information about the cardiovascular activity. In this paper, a new method for estimating systolic and diastolic BP in contact-free for comfort is proposed using a millimeter-wave (MMW) sensor. The MMW sensor is sensitive to small cardiac motion of $1 \mathrm{~mm}$ or less included in chest surface motion because of the higher frequency and wider bandwidth available, thereby continuous systolic and diastolic BP per heartbeat would be able to be estimated from the time-domain features of unique cardiac motion waveform. Measurement was conducted with the MMW sensor system and the estimated systolic and diastolic BP are also compared with an intermittent BP monitor. As a result, the sensor system is found to estimate systolic and diastolic BP continuously.
\end{abstract}

Keywords: Millimeter-wave, continuous blood pressure, monitoring sensor

Classification: Sensing

\section{References}

[1] M. Shibao, A. Kajiwara, "Heart-rate monitoring of moving persons using 79 GHz ultra-wideband radar sensor," IEICE Communications Express, Volume 9 Issue 5 Pages 125-130, 2020.

[2] Y. Uomoto, A. Kajiwara, "Proposal of Heart Rate Estimation Method Using Stepped FM-UWB Sensor," IEEJ Transactions on Electronics, Information and Systems Vol.138, No.7, pp.921-926, July 2018.

[3] I. Yamada, "Physiological information sensing technology aimed at preventive healthcare,” IEICE Fundamentals Review, Vol.12, No.1, pp.30-37, July 2018.

[4] J. Shao, P. Shi, S. Hu, H. Yu, “A Revised Point-to-Point Calibration Approach with Adaptive Errors Correction to Weaken Initial Sensitivity of Cuff-Less Blood Pressure Estimation,” Sensors 2020, April 2020. doi:10.3390/s20082205.

[5] R. Kawasaki, A. Kajiwara, "Continuous Blood Pressure Estimation using 79GHz Band Millimeter Wave Sensor,” IEICE General Conference, Mar. 2021.

[6] N. Shimomura, M. Otsu, A. Kajiwara, "Empirical study of remote respiration monitoring sensor using wideband system," International conference on signal processing communication systems, Dec. 2012. DOI:10.1109/ICSPCS.2012.6507976. 
[7] E. Sasaki, A. Kajiwara, "Multiple respiration monitoring by stepped-FM UWB sensor," IEEE international conference on computational intelligence \& communication technology, Feb. 2015. DOI:10.1109/CICT.2015.157

[8] D. Buxi, J. Redoute, M. Yuce, "Blood pressure using pulse transmit time from bioimpedance and continuous wave radar." Transaction on IEEE Biomedical engineering, Vol.64, No.4, pp.917-927, April 2017.

[9] R. Klabunde, "Cardiovascular physiology concepts, 3rd edition" Wolters Kluwer, 2021. ISBN-13:9781975150075.

\section{Introduction}

Conventional vital signs sensors require body contact, and most of the sensors are intrusive and impede mobility. For example, we may feel uncomfortable when some sensor is attached to our body. The measurement based on stationary state such as electro-cardiogram (ECG) and sphygmomanometer will also give a sense of restraint. Such negative experiences might alter our heart rate and blood pressure. For the last few years, therefore, wireless sensors have been studied for their potential as medical sensors to measure our important vital signs such as heart rate in contact-free [1][2]. Blood pressure (BP) is also one of the basic vital signs which offer valuable medical information about the cardiovascular activity [3]. Especially, continuous BP monitoring in our daily life is being important to improve early detection of cardiovascular disorders, as well as promoting healthcare. However, most automated sphygmomanometers (hereafter referred to as BP sensor) such as cuff-type BP sensor widely used in home measure the BP intermittently, not continuously. Therefore, it is important to realize continuous BP monitoring in contact-free.

Millimeter-wave (MMW) sensor is well known to be sensitive to small cardiac motion of $1 \mathrm{~mm}$ or less because of the higher frequency and wider bandwidth available [2]. The cardiac motion waveform could be related with dynamic BP such as variation of BP because it is a complex physiological signal that is determined by interplay of left ventricular stroke volume, systemic vascular resistance, and vascular compliance [4]. In this paper, a new method for estimating systolic BP (SBP) and diastolic BP (DBP) in contact-free is suggested using the cardiac motion waveform extracted by MMW sensor where it is required to construct the cardiac waveform based features-to-BP translation model prior to the BP monitoring [5]. The model will not change during subsequent long-term BP monitoring, but might preferably be updated with a better estimation accuracy. The continuous BP would be estimated from the chest surface motion waveform including small cardiac motion [1][2]. That is, the chest surface motion is detected using a range-bin diversity method, while the cardiac motion is extracted from the chest surface motion by means of a wavelet-based multi-resolution analysis (MRA) [2]. Therefore, the extraction accuracy would not be affected by any body movements and motions. Next, the time-domain features extracted from the cardiac motion are then translated to systolic BP (SBP) and diastolic BP (DBP) by means of our developed heuristic estimation model (based on linear regression model with a referenced 
Omron BP monitor). From the above approaches, the extracted features from cardiac motion would be able to continuously track the SBP and DBP with promising accuracy. There is no reports regarding the sensor system to realize continuous BP monitoring in contact-free as far as the authors know.

Measurement was conducted with the MMW sensor and estimated continuous SBP and DBP for moving human subjects are compared with the cufftype intermittent BP monitor. As a result, our suggested sensor system is found to be able to estimate SBP and DBP continuously according to the heartbeat.

\section{MMW radar based continuous blood pressure sensor}

Fig. 1 shows the block diagram of continuous BP monitoring sensor where three approaches are used; 1) radar range-bin diversity (range-bin means range-resolution cell), 2) MRA analysis, and 3) signal features-to-BP translation to estimate continuous SBP and DBP.

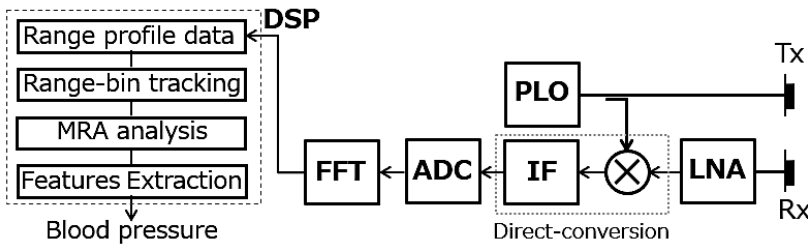

Fig.1 Block diagram of MMW sensor and signal flow diagram

1) Radar range-bin diversity method: Vital signs sensor without restricting any motion and movement is considered desirable. However, the body positioned range-bin of MMW sensor may change during walking. In the MMW sensor, a radar range-bin diversity is employed to track and select the appropriate range-bin signal, which is called range-bin or sampling diversity [6][7], and the sensor system would be applied to various applications such as stress-free and casual health-care monitoring.

2) MRA analysis: The non-stationary cardiac motion waveform included in the received signal is much weaker than the respiratory motion, thereby it is not appropriate to use FFT. In this paper, an MRA is employed to extract the cardiac motion waveform where that orthogonal wavelets are employed [2]. That is, the cardiac motion waveform is reconstructed using detail coefficients to completely separate larger respiratory motion waveform from the received signal. The resulting signal shows cardiac motion which would be unique to each person and his physical condition.

3) Signal features-to-BP translation: The cardiac motion is associated not only with heart rate but also with BP, thereby some time-domain features of cardiac motion waveform would also be related to the SBP and DBP. In this paper, heuristic signal feature-to-SBP translation model has been constructed by means of a simple regression technique with a cuff-type BP monitored values. Similarly, signal feature-to-MBP (mean BP) translation model has been also constructed since it is not easy to find features related to the DBP. From the MBP, DBP can be calculated using the following equation [8]: 


$$
M B P=D B P+\frac{S B P-D B P}{3}
$$

From the above, the cardiac motion waveform would be able to track the SBP, MBP and DBP with promising accuracy. The continuous SBP will also be able to be estimated using a contact-type pulse transmit time (PTT) method which measures the time delay for the BP pulse wave to travel between two arterial sites [9]. However, the method requires complex sensing and analysis circuitry, thereby the related devices is very expensive and inconvenient for our general users.

\section{Measurements}

A. Measurement set-up

The measurement was conducted using a $79 \mathrm{GHz}$-band MMW sensor module with $3.4 \mathrm{GHz}$ bandwidth, $0 \mathrm{dBm}$ transmitted power and $30^{\circ}$ antenna beamwidth. The sensor with a FCM (fast-chirp modulation) architecture transmits a series of 48 FCM waveforms per a frame time of 0.1 second, while the received signal from some object is mixed with the transmitted signal to produce the IF beat which will give the distance and Doppler. The frame time would not be enough to detect the cardiac motion waveform, thereby the $48 \mathrm{FCM}$ waveforms are divided into $12 \mathrm{sub}-$ frames with 4 FCM waveforms. Please note that the substantial frame rate is increased 12 times at the cost of decreasing the signal-noise rate. The resulting IF beat is transformed into time-domain range-profile data using an FFT. Measurement was conducted for subjects under a protocol approved by the University of Kitakyushu and the informed consent was obtained for all subjects.

\section{B. Measurement results}

There are several features for cardiac movement waveform, systolic time (ST), cardiac cycle (CC), and another set of features including the first and second derivative of the cardiac movement [3][8]. In this paper, two features of ST and $\mathrm{CC}$ are considered among the above-mentioned features since the MMW sensor is subject to scattering noise unlike contact-type PPG and ECG. The ST and CC were measured using the MMW sensor placed approximately $1 \mathrm{~m}$ away from for each subject. Fig.2(a) shows scatter plots of estimated features (ST and CC) and measured BPs (SBP and mean BP) for arbitrarily selected two subjects A and B where each regression line for modeling the relationship is also overlaid. It is seen that there are negative considerable correlations between the features and measured BPs. It may be easily comprehensible to see the feature-BP correlation for quantitively evaluating the MMW sensor. Fig.2(b) shows the correlation efficient for arbitrarily selected three subjects. The correlation is very strong at $0.91 \sim 0.96$ for subject $\mathrm{A}$ and $\mathrm{C}$, but not very strong for subject $\mathrm{B}$. The correlation may be improved incorporating another set of features. The correlations mentioned above would therefore be available as the translation models to estimate the continuous BPs with the MMW sensor. 
The demonstration experiment was held for walking subjects A and B using the feature-to-BP translation models. Fig. 3 shows the continuous SBP and DBP for an experimental scenario of subject walking slowly toward the sensor. Continuous BP sensor is not available since it is currently only obtained for medical use. Instead, an arm cuff-type BP monitor has been used. The referenced SBP and DBP values measured by an arm cuff-type BP monitor are also overlayed. Please note that the BP monitor (intermittently measured at intervals between 20 to 30 minutes) can only be used when stationary. It is seen from Fig.3 that the estimated SBP and DBP are in good agreement with the intermittent BP. The measurement was also conducted for the other subjects and the results, which are not shown due to the space limitations, were found to show the same tendency as Fig.3.
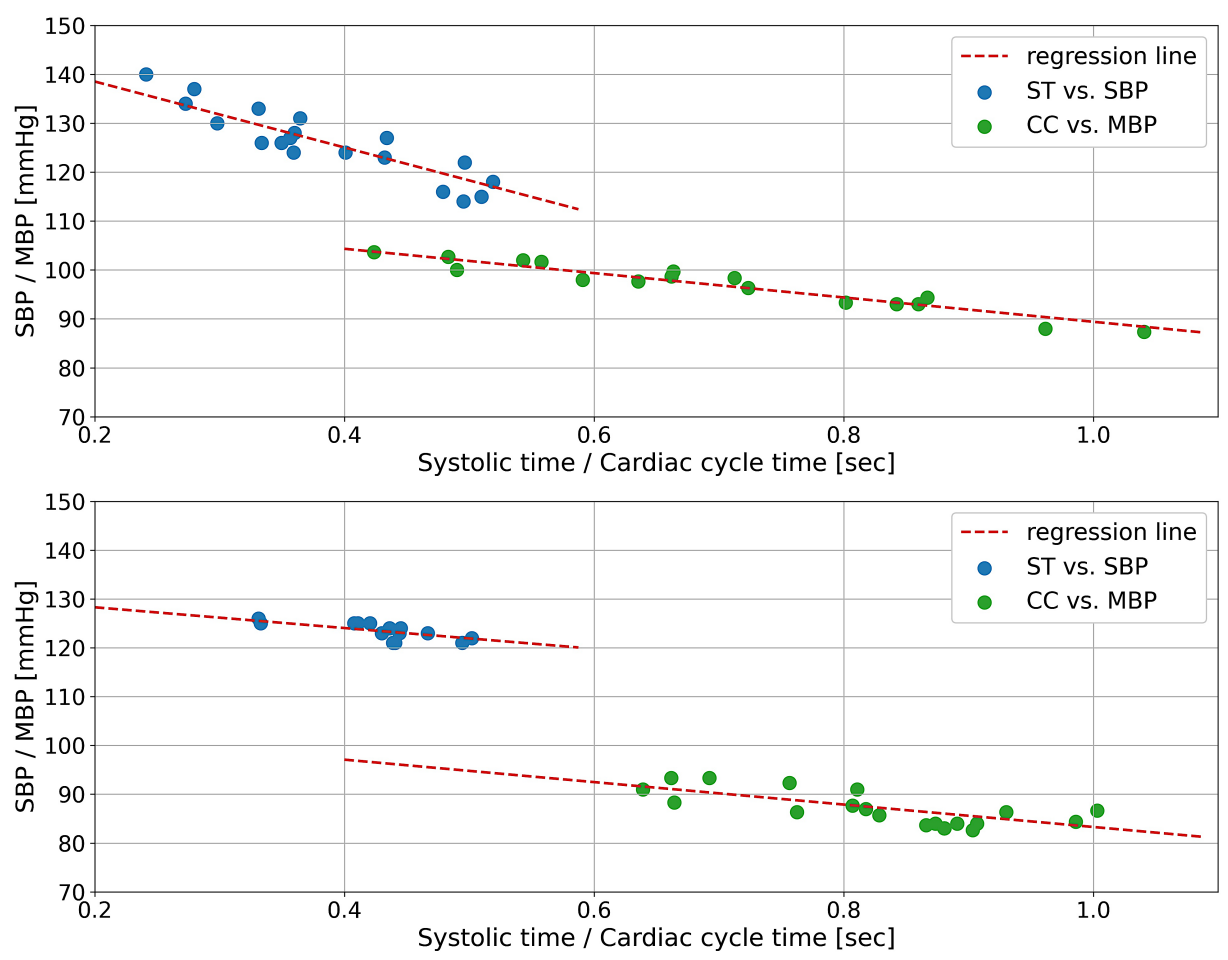

(a) Scatter diagrams of BPs (systolic BP and mean BP) and cardiac movement features (ST and CC). (upper: Subject A, lower: Subject B)

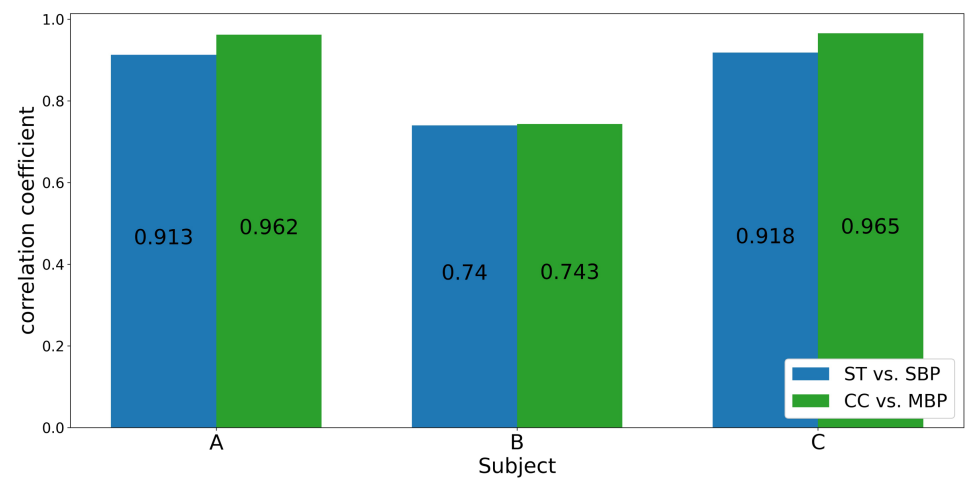

(b) Correlation coefficient for three subjects

Fig.2 Correlation between cardiac movement feature and BP 


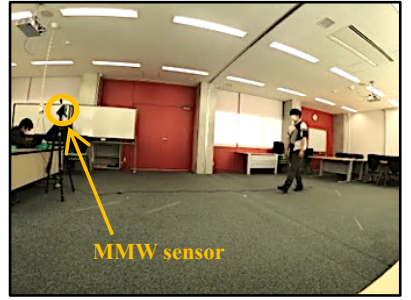

(a) Measurement scene: each subject is walking towards the MMW sensor but resting only while measuring BPs using an arm cuff-type BP monitor because the monitor can only be used when stationary.
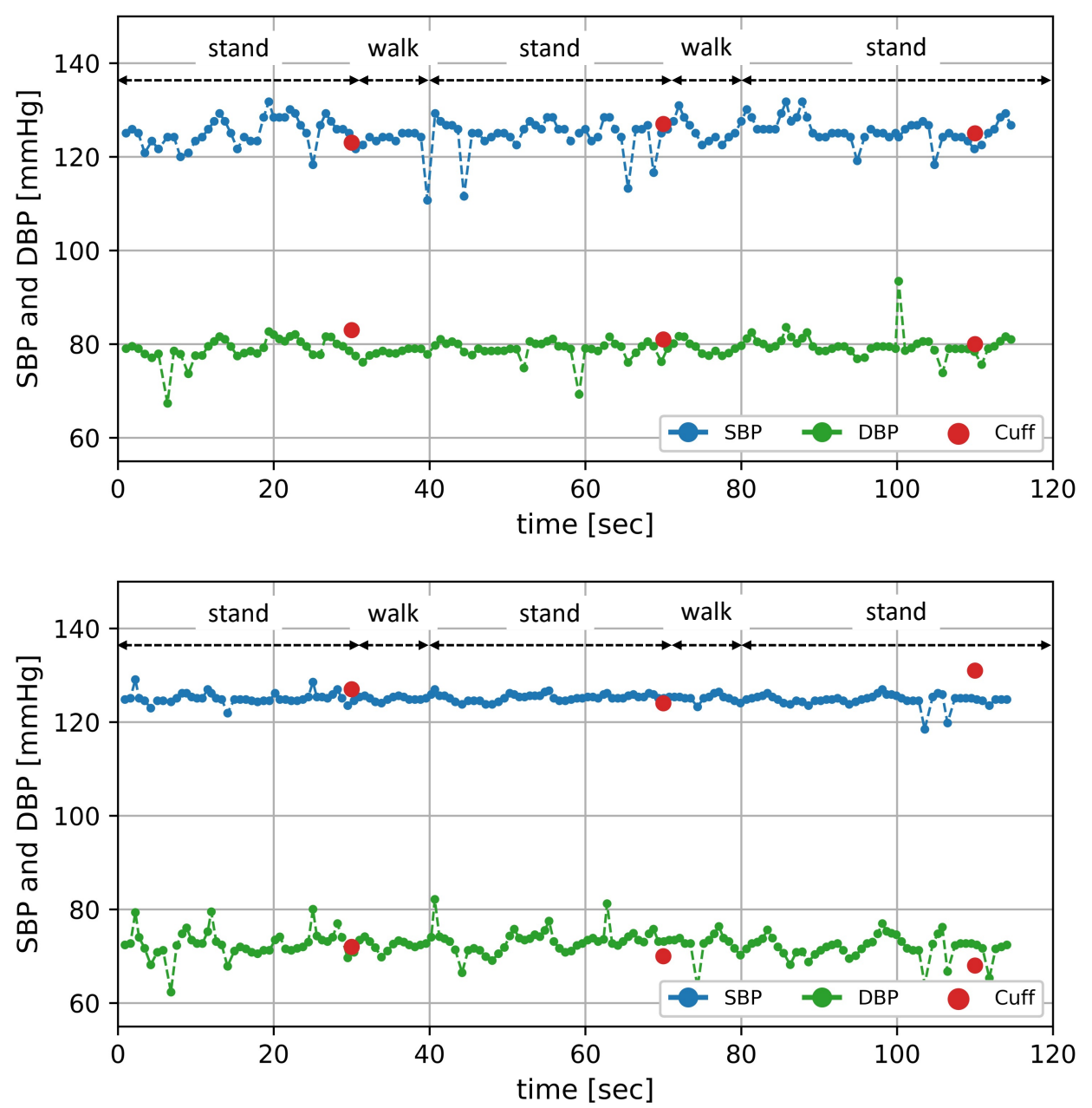

(b) Continuous SBP and DBP values of Subject A(upper) and B(lower)

Fig.3 Continuous BP fluctuation per heartbeat

\section{Conclusions}

This paper has presented a new method for estimating systolic and diastolic BP using only the cardiac movement extracted by an MMW sensor where three approaches of range-bin diversity, MRA analysis, and features of cardiac movement-to-BP translation model are employed. Two-stage measurements were conducted; the first is to establish empirical ST-to-SBP and CC-to-MBP translation models and the second is to investigate the usefulness of continuous BP monitoring with the above translation models. As a result, the features of cardiac movement have the high correlation with BPs and the BP monitoring sensor was found to be useful to estimate continuous systolic and diastolic BP according to heartbeat. 